\title{
Redshift Evolution of the Nonlinear Two-Point Correlation Function
}

\author{
Chung-Pei Ma \\ Department of Physics and Astronomy, University of Pennsylvania, Philadelphia, PA 19104; \\ cpma@strad.physics.upenn.edu
}

\begin{abstract}
This paper presents a detailed theoretical study of the two-point correlation function $\xi$ for both dark matter halos and the matter density field in five cosmological models with varying matter density $\Omega_{\mathrm{m}}$ and neutrino fraction $\Omega_{\nu}$. The objectives of this systematic study are to evaluate the nonlinear gravitational effects on $\xi$, to contrast the behavior of $\xi$ for halos vs. matter, and to quantify the redshift evolution of $\xi$ and its dependence on cosmological parameters. Overall, $\xi$ for halos exhibits markedly slower evolution than $\xi$ for matter, and its redshift dependence is much more intricate than the single power-law parameterization used in the literature. Of particular interest is that the redshift evolution of the halo-halo correlation length $r_{0}$ depends strongly on $\Omega_{\mathrm{m}}$ and $\Omega_{\nu}$, being slower in models with lower $\Omega_{\mathrm{m}}$ or higher $\Omega_{\nu}$. Measurements of $\xi$ to higher redshifts can therefore be a potential discriminator of cosmological parameters. The evolution rate of $r_{0}$ for halos within a given model increases with time, passing the phase of fixed comoving clustering at $z \sim 1$ to 3 toward the regime of stable clustering at $z \sim 0$. The shape of the halo-halo $\xi$, on the other hand, is well approximated by a power law with slope -1.8 in all models and is not a sensitive model discriminator.

To appear in the Astrophysical Journal (Vol. 510, January 1 1999)
\end{abstract}

Subject headings: cosmology : theory - dark matter - elementary particles - galaxies: evolution - large-scale structure of universe - methods: numerical

\section{Introduction}

The two-point correlation function $\xi(r)$ is a fundamental quantity in cosmology. For the density field of matter, it provides the most basic statistical measure of gravitational clustering, and its Fourier pair is simply the power spectrum $P(k)$. For galaxies, $\xi$ offers a convenient measure of their clustering strength, and a determination of $\xi$ is among the most important goals of every major galaxy survey. Although $\xi$ is not independent from $P$, the estimators for the two quantities have different error properties and therefore both carry useful information.

The two-point correlation function of nearby galaxies in physical space has been determined in several galaxy redshift surveys and is found to be well approximated by a power law, $\xi=\left(r / r_{0}\right)^{-\gamma}$, 
over the distance range $1<r<20 h^{-1} \mathrm{Mpc}$. Analysis of the early CfA sample of 2400 galaxies (complete to $m_{B}=14.5$ ) gives a correlation length of $r_{0}=5.4 \pm 0.3 h^{-1} \mathrm{Mpc}$ and a slope of $\gamma=1.77$ for $1 \lesssim r \lesssim 10 h^{-1} \mathrm{Mpc}$ (Davis \& Peebles 1983). Subsequent optical surveys generally have yielded consistent results. The combined CfA2 and Southern Sky Redshift Surveys of 12812 galaxies, for example, give $r_{0}=4.95 \pm 0.13 h^{-1} \mathrm{Mpc}$ and $\gamma=1.73 \pm 0.03$ when cluster galaxies are removed (Marzke et al. 1995). The Las Campanas Redshift Survey (Shectman et al. 1996) of 19558 galaxies is found to have $r_{0}=5.06 \pm 0.12 h^{-1} \mathrm{Mpc}$ and $\gamma=1.862 \pm 0.034$ (Jing, Mo, and Borner 1998). Surveys at other wavelengths have shown larger variations. The 1.2 Jy IRAS survey of 10000 galaxies yields $r_{0}=3.76 h^{-1} \mathrm{Mpc}$ and $\gamma=1.66$ for $r<20 h^{-1} \mathrm{Mpc}$ (Fisher et al. 1994). A small sample of $3001.4 \mathrm{GHz}$ radio sources is found to have $r_{0}=11 h^{-1} \mathrm{Mpc}$ and $\gamma=1.8$, where the enhanced correlations are attributed to the high optical luminosity and rich environments inhabited by radio galaxies (Peacock \& Nicholson 1991).

Although measurements of $\xi(r)$ for nearby galaxies are abundant, the two-point correlation function at higher redshifts is an important quantity that has only begun to be determined. Deep redshift surveys are clearly required for this purpose, and initial results have been somewhat conflicting. The Autofib survey of 1100 galaxies from 33 pencil beams, for example, shows no evidence of evolution, giving $r_{0}=6.2 \pm 0.4 h^{-1} \mathrm{Mpc}$ and $r_{0}=6.8 \pm 0.4 h^{-1} \mathrm{Mpc}$ for the samples below and above the survey median redshift $\langle z\rangle=0.16$, respectively (Cole et al. 1994). The Canada-France Redshift Survey, on the other hand, finds a decreasing correlation function with increasing redshift, with $r_{0}=1.33 \pm 0.09 h^{-1} \mathrm{Mpc}$ at $z=0.53$ (Le Fevre et al. 1996). A sample of 183 field galaxies covering 216 square arcmin from the CNOC (Canadian Network for Observational Cosmology) cluster survey yields $r_{0}=2.2 \pm 0.5 h^{-1} \mathrm{Mpc}$ for $\langle z\rangle=0.37$ (Shepherd et al. 1997). A sample of $248 \mathrm{~K}$-band selected galaxies from two fields of combined area of 27 square arcmin gives $r_{0}=2.9 \pm 0.34 h^{-1} \mathrm{Mpc}$ at $z=0.34, r_{0}=2.0 \pm 0.3 h^{-1} \mathrm{Mpc}$ at $z=0.62$, and $r_{0}=1.4 \pm 0.21 h^{-1} \mathrm{Mpc}$ at $z=0.97$ (Carlberg et al. 1997). The shape of $\xi$ shows little variation with redshift and is well fit by a power law with a slope $\gamma=1.7$ to 1.8 in all surveys. More measurements from deep surveys covering larger areas of the sky will provide more robust determination of $\xi(r)$ beyond $z \approx 0$.

On the theoretical side, models for the redshift evolution of $\xi(r)$ have been simplistic for the most part, and the interpretation of observational results is often cast either in the regime of stable clustering in physical space $\left(\xi \propto a^{3}\right.$, where $\left.a=(1+z)^{-1}\right)$, or fixed clustering in comoving space. Uncertainties and statistical fluctuations in older data sets due to small galaxy numbers and limited survey fields are often too large to allow a finer distinction beyond these two regimes, but data from the ongoing and planned future deep redshift surveys certainly have the potential for discriminating among cosmological parameters, if the evolution of $\xi(r)$ follows distinct patterns in different models. This provides the motivation for the extensive investigation of $\xi$ presented here for a wide range of redshift and cosmological models.

Theoretical predictions of $\xi$ fall in two categories: the density field and dark matter halos. Much work has been done for the former (Jain 1997; Sheth and Jain 1997; Matarrese et al. 1997; 
Jenkins et al. 1998; and references therein), particularly in the context of scale-free models with power-law $P(k)$ and the cold dark matter (CDM) models. No systematic study, however, has been carried out to investigate a spectrum of models with different matter densities as well as neutrino masses over a large redshift range. Theoretical calculations of $\xi$ for the density field can enhance our general understanding of gravitational clustering, but the results cannot be compared directly with the observed $\xi$ without the knowledge of the relationship between the density field and galaxies.

The two-point correlation function of dark matter halos, on the other hand, bears closer resemblance to the observed $\xi$. Analytical bias models have been constructed to relate $\xi$ for the density field to halos (Moscardini et al. 1998), but a more direct, less model-dependent approach is to compute the halo-halo $\xi$ from numerical simulations of structure formation. Since this requires more extensive effort, only a few individual models have been studied. Gelb \& Bertschinger (1994b), Brainerd \& Villumsen (1994), Bagla (1997), and Jing (1998), for example, examined $\xi$ of both matter and halos in the standard CDM model (although with different halo identification schemes. See $\S 3.2$ ). The correlation function in the $\Omega_{\nu}=0.3$ cold + hot dark matter (C+HDM) model has been studied in Klypin et al. (1993) and Jing et al. (1994), but this model has been shown to possess too little small-scale power at high redshifts to account for the amount of observed collapsed objects. Colin et al. (1997) is the only work thus far that has specifically quantified the redshift evolution of halo-halo $\xi$ in different models. Both $\Omega_{\mathrm{m}}=1$ and $\Omega_{\mathrm{m}}=0.2$ CDM models were examined, but their choice of high Hubble constant, $h=1$, exceeds all current observational values and also gives the $\Omega_{\mathrm{m}}=1$ CDM power spectrum a shape parameter of $\Gamma=1$, which is far above $\Gamma \approx 0.25$ indicated by galaxy clustering and the value of $\Gamma=0.5$ for the standard CDM model.

In this paper I present results from an extensive theoretical study of the properties and evolution of $\xi(r)$ in three classes of structure formation models that are of current cosmological interest: CDM, C+HDM, and flat low-density CDM models with a cosmological constant (LCDM). The objectives are to (1) evaluate the nonlinear gravitational effects on $\xi ;(2)$ compare the behavior of $\xi$ for matter vs. halos, which reflects the bias between matter and halos and is sometimes a confused issue in the literature; and (3) quantify the redshift evolution of $\xi$ and its dependence on cosmological parameters. No such systematic study over this wide range of cosmological models and redshift has been carried out thus far. In order to determine the nonlinear evolution of $\xi$, numerical simulations of structure formation in five flat models with matter density $\Omega_{\mathrm{m}}=1,0.5$, 0.3 , and neutrino fraction $\Omega_{\nu}=0.1$ and 0.2 are performed (see $\S 2$ ). Results of $\xi$ for both the density field and halos from linear as well as nonlinear calculations are discussed in $\S 3$. Particular emphasis is placed on quantifying and contrasting the redshift dependence of the halo-halo $\xi$ in different cosmogonies, which can serve as a potential discriminator of cosmological parameters when more high-redshift data are collected. 


\section{Simulations of Nonlinear Clustering}

Numerical simulations are performed to determine the nonlinear two-point correlation functions for both the density field and dark matter halos in five cosmological models that include two C+HDM, two LCDM, and the standard CDM model. For this study to form a controlled numerical experiment, the simulations for the $\mathrm{C}+\mathrm{HDM}$ and LCDM models reported here are all performed in a $(100 \mathrm{Mpc})^{3}$ comoving box with a Plummer force softening length of $50 \mathrm{kpc}$ comoving (for the given value of the Hubble constant in each model), and identical phases are used in the initial conditions for the runs. Two additional large simulations in a $(640 \mathrm{Mpc})^{3}$ box with a force softening length of $160 \mathrm{kpc}$ and a different set of initial conditions are used to test the effects of numerical resolution and realization on our results (see $\S 3$ ). The primordial power spectra for all models have a spectral index of $n=1$, and the density fluctuations are drawn from a random Gaussian distribution. The gravitational forces are computed with a particle-particle particle-mesh $\left(\mathrm{P}^{3} \mathrm{M}\right)$ code (Bertschinger \& Gelb 1991; Gelb \& Bertschinger 1994ab; Ma \& Bertschinger 1994b). The number of simulation particles used to represent the cold dark matter is $256^{3}$ for the $640 \mathrm{Mpc}$ CDM run, and $128^{3}$ for the rest. For the hot dark matter component in C+HDM models, $128^{3}$ and $10 \times 128^{3}$ particles are used in the $\Omega_{\nu}=0.1$ and 0.2 models, respectively. Although the latter is needed for a fine sampling of the neutrino velocity phase space (Ma \& Bertschinger 1994a), the two-point correlation function - the subject of study here - mostly probes the spatial distribution of matter. Tests with a reduced number of hot particles have been performed in the $\Omega_{\nu}=0.2$ model to verify that $128^{3}$ is sufficient for an accurate determination of $\xi$.

For the C+HDM models, only $\Omega_{\nu}=0.1$ and $\Omega_{\nu}=0.2$ are studied here because structure forms too late in models with $\Omega_{\nu}>0.2$ to account for high-redshift galactic structures (Ma et al. 1997 and references therein). A baryon fraction of $\Omega_{\mathrm{b}}=0.05$ and a Hubble constant of $h=0.5$ are assumed, and the cold dark matter fraction is $\Omega_{\mathrm{cdm}}=0.85$ and 0.75 for the two models, respectively. The rms linear mass fluctuation in spheres of radius $8 h^{-1} \mathrm{Mpc}$ is $\sigma_{8}=0.9$ and 0.82 for $\Omega_{\nu}=0.1$ and 0.2, when the 4-year COBE result (Bennett et al. 1996; Gorski et al. 1996) is used as the normalization.

The two LCDM models chosen for the simulations have $\left(\Omega_{\mathrm{m}}, \Omega_{\Lambda}, h\right)=(0.3,0.7,0.75)$ and $(0.5,0.5,0.7)$. The parameter $\Gamma=\Omega_{\mathrm{m}} h$ characterizing the shape of the power spectrum is 0.23 and 0.35 for the two models. Both models are COBE-normalized, with $\sigma_{8}=1.28$ and 1.53, respectively. The COBE-normalized standard CDM model with $\Omega_{\mathrm{m}}=1$ and $h=0.5$ (no baryons) is also included in this study as a point of standard reference despite of its excess power on small scales. The corresponding $\sigma_{8}=1.4$ is a factor of $\sim 2$ higher than that required by cluster abundance, but the results quoted here can be easily translated to a lower normalization by rescaling the redshift. The results for the standard CDM model are computed from a $\mathrm{P}^{3} \mathrm{M}$ simulation performed by Gelb \& Bertschinger (1994ab), which employed a numerical algorithm and numerical parameters similar to the LCDM and C+HDM runs used here: $66 \mathrm{kpc}$ for the Plummer force softening distance, 100 Mpc box, and $144^{3}$ particles. The baryon fraction is set to zero for both LCDM and the standard CDM models so that the effects of changing $\Omega_{\mathrm{m}}$ can be clearly studied. 


\section{Two-Point Correlation Function}

The two-point correlation functions $\xi$ for the mass density field and dark matter halos have different shapes and follow distinct evolutionary histories. They are discussed separately in $\S 3.1$ and $\S 3.2$. Results for the evolution of the correlation length are discussed in $\S 3.3$. All figures for $\xi$ are presented in physical coordinates $r$, but its behavior in comoving space is also discussed.

\section{1. $\quad \xi$ of the Density Field}

The two-point correlation function of the density field measures the lowest-order gravitational clustering of matter in a given cosmological model. It is the Fourier transform of the power spectrum $P(k)$ for the density fluctuation $\delta \rho / \rho$, and can be written as

$$
\xi(r)=\int_{0}^{\infty} \frac{d k}{k} 4 \pi k^{3} P(k) \frac{\sin k r}{k r} .
$$

In the linear regime, $\xi$ is easily calculated from the linear $P(k)$, which can be obtained from either direct integration of the coupled Boltzmann and Einstein equations, or analytical approximations for various cosmological models that have been published in the literature (e.g., Bardeen et al. 1986; Efstathiou et al. 1992; Ma 1996). In the nonlinear regime, $\xi$ can be calculated either from the particle positions in numerical simulations directly, or from integration of the few published fitting functions for the nonlinear $P(k)$. For the nonlinear $P(k)$, Hamilton et al. (1991) examined scale-invariant models with power-law spectra, Jain et al. (1995) and Peacock \& Dodds (1996) studied the flat and open CDM models, while Ma (1998) provided a general approximation valid for CDM, LCDM, as well as C+HDM models.

Figures 1 to 3 compare $\xi(r)$ for the linear and nonlinear density fields at various redshifts for the five models chosen for this study - the standard CDM (Fig. 1), the $\Omega_{\mathrm{m}}=0.5$ and $0.3 \mathrm{LCDM}$ models (Fig. 2), and the $\Omega_{\nu}=0.1$ and $0.2 \mathrm{C}+\mathrm{HDM}$ models (Fig. 3). The dashed curves show the linear $\xi$ computed from equation (1). For the CDM and LCDM models, the input linear $P(k)$ is the Bardeen et al. fitting function (1986) with the shape parameter $\Gamma=\Omega_{\mathrm{m}} h$ (no baryons are assumed in these models). For the C+HDM models, the input is the fitting function of Ma (1996) for the density-averaged linear $P(k)$, where $P(k)=\left[\Omega_{\nu} P_{\nu}^{1 / 2}+\left(1-\Omega_{\nu}\right) P_{c}^{1 / 2}\right]^{2}$ measures the total gravitational fluctuations contributed by the separate hot and cold components.

The circles in Figures 1 to 3 show the nonlinear $\xi$ calculated directly from the particles in the $(100 \mathrm{Mpc})^{3}$ simulations described in $\S 2$. As a test of the robustness of our numerical results, the solid squares in the bottom panels of Figures 1 and 2 compare $\xi$ computed from different simulations of the CDM and the $\Omega_{\mathrm{m}}=0.3$ LCDM models. Both simulations are performed in a much larger box $-(640 \mathrm{Mpc})^{3}$ in comoving volume - and a force softening distance of $160 \mathrm{kpc}$ (i.e., $80 h^{-1} \mathrm{kpc}$ for CDM and $120 h^{-1} \mathrm{kpc}$ for LCDM). The CDM simulation uses 16.8 million

particles while the LCDM has 2.1 million. As expected, the squares fall slightly below the circles 
at small separation due to the larger force softening in the big-box simulations, whereas the circles fall below the squares at comoving separation $x \gtrsim 10 h^{-1} \mathrm{Mpc}$ due to their smaller box size. In the intermediate regime, however, the results agree well between the two runs that have very different particle numbers, box sizes, and force resolution. This demonstrates that our determination of $\xi$ is robust within the resolution range of the simulations.

As discussed earlier, the nonlinear $\xi$ can also be computed from equation (1) if the corresponding nonlinear power spectrum is known. The solid curves in Figures 1 to 3 show the results for all five models when the analytical approximation of Ma (1998) for the nonlinear $P(k)$ is used as input in equation (11). The results agree well with the nonlinear $\xi$ computed directly from the simulation particles, providing a consistency check.

As Figures 1-3 illustrate, the effects of nonlinear gravitational clustering drastically change the shape of the linear $\xi$ below about $5 h^{-1}$ comoving Mpc. At separations of $\sim 0.1 h^{-1}$ comoving $\mathrm{Mpc}$, the nonlinear contribution boosts the linear correlation amplitude by more than one order of magnitude. As a result, the nonlinear effect on the shape of $\xi$ is to straighten up the linear curves partially, but as the solid curves show, the nonlinear $\xi(r)$ is far from being a simple power law. In particular, $\xi$ acquires a steeper slope in the intermediate scale around $1 h^{-1}$ comoving $\mathrm{Mpc}$ in all five models. Such a change of slope is consistent with similar behavior in the nonlinear power spectrum in Hamilton et al. (1991), Jain et al. (1995), and Ma (1998). It is also reported in scale-free and CDM models by Padmanabhan (1996), Jain (1997), and Jenkins et al. (1998). This non-power-law behavior of $\xi$ of the density field, however, should not be viewed as an inconsistency with observations. The $\xi$ computed from halos discussed in the next section bears closer reality to the observed $\xi$ for galaxies, and as will be shown, the shape of halo-halo $\xi$ is indeed well approximated by a power law.

In the intermediate range between 1 and $10 h^{-1}$ comoving Mpc, Figures 1 to 3 show the interesting feature that the nonlinear $\xi$ dips below the linear $\xi$ before rising rapidly above it on smaller scales. The decrement is present only at $z \lesssim 1$, but it becomes more prominent and extends over a larger range of $r$ as time goes on. This feature is not due to numerical artifacts in the simulations (such as small box size or statistical fluke) because it is seen in all models, in both $100 \mathrm{Mpc}$ and $640 \mathrm{Mpc}$ simulations with different resolution and realization, and in both $\xi$ computed from the particles (i.e. circles and squares) and that computed from integration of the analytical nonlinear $P(k)$ (i.e. solid curves). Mathematically, despite the fact that the nonlinear $P(k)$ is always larger than or equal to the linear $P(k)$ (within the $<10 \%$ fitting error of Ma (1998)), the nonlinear $\xi$ can fall below the linear $\xi$ for a limited range of $r$ because the factor $\sin (k r) / k r$ in equation (11) is oscillatory. Physically, the decrement may reflect the depletion of matter on quasi-linear scales that is fueling the process of gravitational infalls in more clustered regions on smaller scales. The decrement also implies that the nonlinear correlation lengths evolve more slowly than the linear predictions, which contributes to the slower evolution of the halo-halo $\xi$ to be discussed in the next section. This interesting feature may warrant further analytical study. 
The dependence of $\xi(r)$ on the expansion factor $a$ is related to the growth rate of $P(k)$, a well understood phenomenon in the linear regime. For the standard CDM model, the linear $P$ and $\xi$ both grow as $a^{2}$. For $\mathrm{C}+\mathrm{HDM}$ models, the linear $P$ and $\xi$ also increase as $a^{2}$ above the neutrino free-streaming scale, but the growth is retarded below this scale because the phase-mixing of neutrinos reduces the density perturbations. An accurate analytic approximation (with $<10 \%$ error) for this scale-dependent effect is given by equation (11) of Ma (1996). For critically-flat LCDM models, the linear $P$ and $\xi$ again grow as $a^{2}$ until the Universe becomes $\Lambda$-dominated at $1+z \approx \Omega_{\mathrm{m}}^{-1 / 3}$. After this epoch, the growth is slowed down (Heath 1977). However, unlike the $\mathrm{C}+\mathrm{HDM}$ models in which the neutrino free-streaming imprints a characteristic scale, the retardation of the linear growth rate in the LCDM model is independent of length scales. In the nonlinear regime, the evolution of $\xi$ for the density field in the CDM model has been discussed in Jain (1997) and Matarrese et al. (1997).

\subsection{Halo-Halo $\xi(r)$}

In order to determine the correlations of the simulated halos, the Denmax algorithm (Gelb \& Bertschinger 1994ab; Ma \& Bertschinger 1994b; Frederic 1995ab) is first used to identify dark matter halos in the output of the simulations. The Denmax algorithm is designed to associate particles with density peaks by moving the particles along the gradient of the mass density field until they settle into the peaks. The density field is first computed on a very fine grid from the particle positions using the triangular-shaped cloud interpolation scheme (Hockney \& Eastwood 1988), and then convolved with a Gaussian filter to remove grid effects. After the particles are associated with density peaks by Denmax, the energy of the particles in each halo is computed and the unbound ones are removed. An overdensity of 200 is also imposed to select virialized halos that are potential galaxy hosts. Due to the small smoothing length required for identifying galactic-size halos (typically 1/1000th the box length), this method is more CPU-intensive than the friends-of-friends (FOF) algorithm used in previous numerical studies of halo-halo correlation functions (e.g., Brainerd \& Villumsen 1994; Colin et al. 1997). The Denmax algorithm is chosen here, however, because extensive tests have demonstrated that it generally identifies distinct density concentrations more reliably than the FOF algorithm, for which an arbitrary linking length must be assigned (Gelb \& Bertschinger 1994ab; Frederic 1995ab).

All dissipationless simulations suffer to some degree the overmerging problem in regions of high overdensities. When individual dark matter halos merge in these simulations, they form a larger, smooth halo with little substructure due to the lack of dissipation. The extent of this problem can be quantified by examining output of hydrodynamic simulations, which are designed to follow both the dark matter and a gas component that is allowed to cool and condense to high densities. Massive dark matter halos of the size of galaxy clusters in these simulations have been seen to host multiple subclumps of condensed gas - presumably the sites of galaxies - that had survived the merger due to their high densities and small cross sections (e.g., Katz et al. 1992; 
Evrard et al. 1994). It is not easy to identify galactic halos in such highly clustered regions from the dark matter distribution alone, and various methods have been proposed to locate galactic sites in clusters in $\mathrm{N}$-body simulations. However, tests against hydrodynamical simulations have so far indicated that no method proves satisfactory on cluster scales (Summers et al. 1995).

Outside of the dense cluster environment, on the other hand, dark matter halos identified by reliable algorithms in $N$-body simulations have been found to give robust predictions for galaxy clustering statistics. For the two-point correlation function, for example, Summers et al. (1995) found good agreement between $\xi$ determined from dark matter halos and from the gas in hydrodynamical simulations on scales above $1 \mathrm{Mpc}$, and most halo identification methods gave consistent results (see their Fig. 12). Gelb \& Bertschinger (1994b) studied two break-up methods that were designed to reconstruct the galactic substructure in clusters by splitting the massive halos in $N$-body simulations into multiple sub-halos. The amplitude of the two-point correlation was indeed boosted significantly on scales below $1 \mathrm{Mpc}$ due to the break-up procedure. On scales above $1 \mathrm{Mpc}$, however, both the shape and the correlation length were hardly changed whether the massive halos were split up or not (see their Figs. 9 and 10). These studies all indicate that the overmerging problem in $N$-body simulations is confined to scales of $\sim 1$ comoving Mpc and below; it therefore does not affect the determination of the correlation length (discussed in the next section). Moreover, most measurements of $\xi$ are based on surveys of galaxies in blank fields devoid of massive clusters (e.g. Shepherd et al. 1997; Le Fevre et al. 1996; Carlberg et al. 1997). The problem with galaxy identification in the cluster environment therefore should not be of serious concern to this study.

Figures 4-6 show the resulting two-point correlation functions of the resolved halos determined from our simulations. Five models - the standard CDM (Fig. 4), the $\Omega_{\mathrm{m}}=0.3$ and 0.5 LCDM models (Fig. 5), and the $\Omega_{\nu}=0.1$ and $0.2 \mathrm{C}+\mathrm{HDM}$ (Fig. 6) - are shown for comparison. For each model, the figures illustrate the redshift evolution by plotting $\xi$ from $z=0$ up to 4 . The left and right panels are computed from virialized halos in two mass ranges, $M>10^{11.5} M_{\odot}$ and $M>10^{12.5} M_{\odot}$, respectively. These two different mass limits are chosen to illustrate the dependence of $\xi$ on halo populations. The figures show that the more massive halos have a higher correlation amplitude and length. Since the observed $\xi$ is determined from field galaxies not associated with massive clusters (as discussed above), I have tested the effects of massive halos on the determination of $\xi$ by imposing an upper mass cut of $M<10^{14} M_{\odot}$. Due to the rarity of these high-mass objects, $\xi$ is found to depend little on whether these objects are included in the sample or not. For simplicity, such an upper mass cut will therefore not be imposed in the results reported here.

As Figures 4-6 show, the shapes of the halo-halo $\xi$ do not depend noticeably on the models or the halo mass cuts. In contrary to $\xi$ for the density field (Figs 1 to 3), the halo-halo $\xi$ is well approximated by a power law with the observed slope of $\sim-1.8$ between 1 and $10 h^{-1}$ comoving Mpc. On scales of below $\sim 1 h^{-1}$ comoving Mpc, $\xi$ starts to flatten out as $z$ approaches 0 in the CDM and $\Omega_{\mathrm{m}}=0.5 \mathrm{LCDM}$ models that have relatively large small-scale power. This 
behavior largely reflects the over-merging problem in dissipationless simulations discussed above. The flattening of $\xi$ disappears when either halo break-up procedures are applied (e.g., Gelb \& Bertschinger 1994b) or gas particles are used as galaxy tracers. Since the shape of $\xi$ carries little information about the underlying cosmogony, no attempts will be made here to correct for the flattening of $\xi$ on small scales. Instead, the focus of this study is on the more interesting quantity, the correlation length and its evolution. They are determined by the reliable part of $\xi$ at $r>1 h^{-1}$ $\mathrm{Mpc}$ and are largely unaffected by the overmerging problem.

\subsection{Redshift Evolution of $\xi$ and $r_{0}$}

Comparison of the two-point correlation function for halos in Figures 4-6 to that for the density field in Figures 1-3 shows that the evolution of the halo-halo $\xi$ is markedly slower than the matter density field in all models. Relative to the density field, the clustering amplitude of halos is higher at $z \sim 4$, but it invariably drops below that of the density field by $z=0$ due to the slow evolution. Such "anti-biasing" effect has been noted in previous studies of the CDM model (Gelb \& Bertschinger 1994b; Colin et al. 1997); the present study shows that this effect occurs in LCDM and $\mathrm{C}+\mathrm{HDM}$ models as well. It is attributed to the continuous disruption and mergers of halos at late times that reduce the clustering of halos to below that of the mass. The relationship between the density field and the halos shows complicated dependence on both length and time scales and on cosmological parameters. Care must therefore be taken not to compare $\xi$ for the density field to the observed $\xi$ since the former does not reflect directly the observed clustering properties of galaxies.

The evolution of $\xi$ for halos depends strongly on the cosmological parameters. Figure 7 shows the redshift dependence of the physical correlation length $r_{0}(z)$ for the simulated halos for two mass ranges in all five models. The fastest growth occurs in the pure CDM model, in which the physical correlation length $r_{0}$ increases by a factor of 8 from $z=4$ to 0 . The correlation length changes more mildly in the LCDM and C+HDM models. For instance, $r_{0}$ increases by a factor of 3 from $z=3$ to 0 in the $\Omega_{\nu}=0.2$ model and a factor of 5.4 from $z=4$ to 0 in the $\Omega_{\mathrm{m}}=0.3 \mathrm{LCDM}$ model. When expressed in comoving coordinates, the halo-halo $\xi$ in fact decreases with time at higher redshifts and is seen to rise only at a later time (see also Brainerd and Villumsen 1994; Bagla 1997). However, one should be careful not to interpret this behavior as actual decrease in the physical clustering amplitude at early times: as Figures 4 to 7 clearly illustrate, the physical $r_{0}$ and $\xi(r)$ always increase monotonically with time in all models. Instead, the decrease merely reflects the fact that the halo clustering rate is slower than the state of fixed clustering in comoving coordinates early on. It eventually surpasses fixed comoving clustering when the comoving correlation length begins to increase (see Fig. 8 below).

The top panels of Figure 7 illustrate that $r_{0}$ increases more slowly in models with smaller $\Omega_{\mathrm{m}}$. This feature is consistent with the slower growth of structure in low-density models. The lower panels compare different $\mathrm{C}+\mathrm{HDM}$ models and indicate that larger $\Omega_{\nu}$ models show slower 
evolution. This can be explained by the slower growth due to the large neutrino free-streaming effects in high- $\Omega_{\nu}$ models. The left and right panels of Figure 7 illustrate that $r_{0}$ also depends on the minimal halo mass selected for the sample, and is larger for the more massive halos (right panels) due to their high clustering amplitudes.

To quantify the evolution of the two-point correlation function with redshift, I find it most convenient to parameterize the physical and comoving correlation lengths directly as

$$
\begin{aligned}
& r_{0}(z)=(1+z)^{-\eta(z)} r_{0}(0), \\
& x_{0}(z)=(1+z)^{-\eta(z)+1} x_{0}(0) .
\end{aligned}
$$

The parameter $\eta$ itself increases with time because as Figure 7 illustrates, the slope of $r_{0}(z)$ generally steepens as $z$ decreases, and the rate of change depends on $\Omega_{\mathrm{m}}$ and $\Omega_{\nu}$. The form of equation (2) is chosen to improve the power-law parameterization commonly used in the literature, where both the shape and redshift dependence of $\xi$ are written as power laws with exponents $\gamma$ and $\epsilon$ :

$$
\begin{aligned}
\xi(r, z) & =\left(\frac{r}{r_{0}(z)}\right)^{-\gamma} \\
\xi(r, z) & =(1+z)^{-3-\epsilon} \xi(r, 0),
\end{aligned}
$$

and $r$ is the pair separation in physical coordinates. It follows from equation (3) that the physical and comoving correlation length evolve as

$$
\begin{aligned}
& r_{0}(z)=(1+z)^{\frac{-3-\epsilon}{\gamma}} r_{0}(0), \\
& x_{0}(z)=(1+z)^{\frac{-3-\epsilon+\gamma}{\gamma}} x_{0}(0) .
\end{aligned}
$$

As Figure 7 illustrates, however, the power-law redshift dependence in equation (đ) does not hold over the redshift range 0 to 4 for any model. Moreover, since the parameter $\epsilon$ in equation (3) was introduced to characterize the redshift evolution of $\xi$ and not $r_{0}$, the evolution of $r_{0}$ depends inconveniently on both $\epsilon$ and $\gamma$. The simple form of equation (2) will therefore be used here. To translate $\eta$ into the parameter $\epsilon$ used in the literature, use

$$
\epsilon=\gamma \eta-3
$$

Some useful theoretical predictions for the evolution of $\xi$ are: (1) The linear theory predicts $\xi \propto(1+z)^{-2}$ for the standard CDM model, corresponding to $\epsilon=\gamma-1$, or $\epsilon \approx 0.8$ and $\eta \approx 2.1$ for $\gamma \approx 1.8 ;(2)$ clustering fixed in comoving coordinates corresponds to $x_{0}(z)=x_{0}(0)$, implying $\eta=1$, or $\epsilon \approx-1.2$; (3) stable clustering in physical space corresponds to $\xi \propto(1+z)^{-3}$, and therefore $\epsilon=0$, or $\eta \approx 1.7$.

Figure 8 shows $\eta(z)$ for the simulated halos in our study. The rise of $\eta(z)$ with time indicates that the halo correlation length $r_{0}$ grows faster than a simple power law with a constant $\epsilon$ assumed 
in the literature (as seen in Figure 7). The parameter $\eta(z)$ also depends on both cosmological parameters $\Omega_{\mathrm{m}}$ and $\Omega_{\nu}$. The label on the right along the vertical axis marks the corresponding $\epsilon$ when $\gamma=1.8$ is assumed for the slope of $\xi$. When $\eta$ crosses unity in a given model, it indicates that gravitational clustering has become faster than no evolution in comoving space (i.e., $\left.x_{0}(z)=x_{0}(0)\right)$. This epoch corresponds to the turn-around redshift at which the initially falling $\xi$ first starts to increase in comoving space. At $z \approx 0$, the CDM, $\Omega_{\mathrm{m}}=0.5 \mathrm{LCDM}$, and $\Omega_{\nu}=0.1$ $\mathrm{C}+\mathrm{HDM}$ models all approach the stable clustering regime of $\eta \approx 1.7$, whereas the $\Omega_{\mathrm{m}}=0.3$ LCDM model exhibits less rapid evolution of $\eta \approx 1$.3. Stable clustering has also been seen for the density field on small scales in the pure CDM model and models with scale-free spectra (Jain 1997). Our study here shows that halos also approach stable clustering in high- $\Omega_{\mathrm{m}}$ models.

The only detailed numerical study of the evolution of halo-halo $\xi$ thus far is Colin et al. (1997), in which the $\Omega_{\mathrm{m}}=1$ and $\Omega_{\mathrm{m}}=0.2$ CDM models are examined. But as discussed in $\S 1$, the $\Omega_{\mathrm{m}}=1 \mathrm{CDM}$ results reported here cannot be compared directly to theirs because of their choice of high Hubble constant $h=1$ and large value of the shape parameter $\Gamma=1$. Since the standard CDM model with $\Gamma=0.5$ used in this paper already suffers from the well-known excess small-scale power, a model with $\Gamma=1$ would produce even more power and worsen the problem. This may be the main cause of the steep slope $\gamma \approx 2.2$ to 2.4 for $\xi$ in Table 2 of Colin et al., which is significantly larger than the value of $\gamma \approx 1.8$ found here and in other works. One should also note that because $r_{0}(z)$ depends on both $\gamma$ and $\epsilon$ (see eq. [4]), their high values of $\gamma$ lead to the large positive $\epsilon$ in their Figure 9 (where $\epsilon$ ranges from $\sim 0$ at $z=5$ to 1.5 at $z=1$ ). When expressed in terms of the parameter $\eta$ introduced earlier, their large values of $\gamma$ and $\epsilon$ do cancel out to some extent, giving $\eta \approx 1.3$ at $z=5$ and 1.87 at $z=0$, but it is still significantly larger than our $\epsilon$ in Figure 8.

\section{Conclusions and Discussion}

Motivated by the extensive observational effort toward determining the evolution of the two-point correlation function $\xi(r)$, I have presented a detailed theoretical study of the the redshift dependence of $\xi$ in five cosmological models with varying mass density $\Omega_{\mathrm{m}}$ and neutrino fraction $\Omega_{\nu}$. The evolution is found to be much more intricate than the simple power-law parameterization of $\epsilon$ commonly assumed in the literature, and a redshift-dependent variable $\eta$ is introduced to quantify the evolution of the correlation length: $r_{0}(z)=(1+z)^{-\eta(z)} r_{0}(0)$. The value of $\eta(z)$ depends strongly on $\Omega_{\mathrm{m}}$ and $\Omega_{\nu}$, and is smaller in models with lower $\Omega_{\mathrm{m}}$ or higher $\Omega_{\nu}$ (Fig. 8), indicating a slower growth in $r_{0}$. Within a given model, $\eta$ increases with time, passing the phase of fixed comoving clustering $(\eta=1)$ at $z \sim 1$ to 3 toward stable clustering $(\eta \approx 1.7)$ at $z \sim 0$.

Although the two-point correlation function $\xi$ provides a fundamental statistical measure of gravitational clustering, care must be taken in specifying the underlying quantity that is being measured and in including the possible nonlinear effects. This study has demonstrated that $\xi$ for the matter density field and dark matter halos have different shapes and evolve with different 
rates. While the local slope of $\xi$ for the nonlinear density field changes with length scales (Figs. 1 to 3 ), the shape of the halo-halo $\xi$ is well approximated by a power law with the observed slope of $\sim-1.8$ independent of cosmological parameters (Figs. 4 to 6 ). It is therefore the redshift evolution of the amplitude of $\xi$ that will reveal the most about cosmogonies. Since halos generally correspond to galaxy sites (except in dense cluster environment), $\xi$ for halos in simulations is a reasonable quantity to compare with observations. The correlation function for the density field provides important insight for theoretical studies of gravitational clustering, but it bears little direct relevance to the observed clustering of galaxies.

On the observational side, ongoing deep redshift surveys of galaxies that cover larger areas of the sky should soon yield more robust determination of $\xi$ beyond $z \approx 0$. Photometric redshifts determined from multi-color surveys and the two-point angular correlation function also offer complimentary measurements of $\xi$ (Connolly, Szalay, \& Brunner 1998; Postman et al. 1998; and references therein). We are currently analyzing galaxy clustering properties in two redshift surveys conducted with the Norris Spectrograph on the Palomar 200-inch telescope (Small et al. 1998). The combined surveys have more than 1000 galaxies in $0.2<z<0.5$. The implications of the theoretical results presented here will be discussed in Small et al. (1998).

Although the evolution of $\xi$ has already been counted as yet another strike against $\Omega_{\mathrm{m}}=1$ models (Carlberg et al. 1997), it must be borne in mind that the nature of high-redshift galaxies has only begun to be unravelled, and that little is known about their dark matter halos. Due to the large uncertainties in the current observational results and theoretical understanding in galaxy evolution, this paper has chosen to investigate the evolution of $\xi$ using simulated dark matter halos within well defined mass ranges. It is nonetheless conceivable that measurements of $\xi$ at higher redshifts are probing different galaxy populations that are not fair counterparts of the local galaxies. Based on future observations, one should be able to refine the theoretical predictions made in this paper by incorporating different dark matter halo populations at higher redshifts.

The author thanks Jim Frederic and Todd Small for valuable discussions. Supercomputing time was provided by the National Scalable Cluster Project at the University of Pennsylvania and the National Center for Supercomputing Applications. 


\section{REFERENCES}

Bagla, J. S. 1997, astro-ph/9711081

Bardeen, J. M., Bond, J. R., Kaiser, N., \& Szalay, A. S. 1986, ApJ, 304, 15

Bennett, C. L. et al. 1996, ApJ, 464, L1

Bertschinger, E., \& Gelb, J. M. 1991, Comput. Phys., 5, 164

Brainerd, T. G. \& Villumsen, J. V. 1994, ApJ, 431, 477

Carlberg, R. G., Cowie, L., Songaila, A., \& Hu, E. 1997, ApJ, 484, 538

Cole, S., Ellis, R., Broadhurst, T., \& Colless, M. 1994, MNRAS, 267, 541

Colin, P., Carlberg, R. G., \& Couchman, H. M. P. 1997, ApJ, 490, 1

Connolly, A. J., Szalay, A. S., \& Brunner, R. J. 1998, ApJ, 499, L125

Davis, M., \& Peebles, P. J. E. 1983, ApJ, 267, 465

Efstathiou. G., Bond, J. R., White, S. D. M. 1992, MNRAS, 258, 1(p)

Evrard, A. E., Summers. F. J., \& Davis, M. 1994, ApJ, 422, 11

Fisher, K. B., Davis, M., Strauss, M. A., Yahil, A., \& Huchra, J. 1994, MNRAS, 266, 50

Frederic, J. J. 1995a, ApJS, 97, 259

Frederic, J. J. 1995b, ApJS, 97, 275

Gelb, J. M. \& Bertschinger, E. 1994a, ApJ, 436, 467

Gelb, J. M. \& Bertschinger, E. 1994b, ApJ, 436, 491

Gorski, K. M. et al. 1996, ApJ, 464, L11

Hamilton, A. J. S., Kumar, P., Lu, E., \& Matthews, A. 1991, ApJ, 374, L1

Heath, D. J. 1977, MNRAS, 179, 351

Hockney, R. W., \& Eastwood, J. W. 1988, Computer Simulations Using Particles (New York: Adam-Hilger)

Jain, B. 1997, MNRAS, 287, 687

Jain, B., Mo, H. J., \& White, S. D. M. 1995, MNRAS, 276, L25

Jenkins, A. et al. 1998, ApJ, 499, 20

Jing, Y. P. 1998, astro-ph/9805202

Jing, Y. P., Mo, H. J., \& Borner, G. 1998, ApJ, 494, 1

Jing, Y. P., Mo, H. J., Borner, G., \& Fang, L. Z. 1994, A\&A, 284, 703

Katz, N., Hernquist, L., \& Weinberg, D. H. 1992, ApJ, 399. L109

Klypin , A., Holtzman, J., Primack, J., and Regos, E. 1993, ApJ, 416, 1

Le Fèvre, O., Hudon, D., Lilly, S., Crampton D., Hammer, F. \& Trees, L. 1996, ApJ, 461, 534 
Ma, C.-P. 1996, ApJ, 471, 13

Ma, C.-P. 1998, submitted to ApJ

Ma, C.-P., \& Bertschinger, E. 1994a, ApJ, 429, 22

Ma, C.-P., \& Bertschinger, E. 1994b, ApJ, 434, L5

Ma, C.-P., Bertschinger, E., Hernquist, L., Weinberg, D. H., \& Katz, N. 1997, ApJ, 484, L1

Marzke, R. O., Geller, M. J., da Costa, L. N., \& Huchra, J. P. 1995, AJ, 110, 477

Matarrese, S., Coles, P., Lucchin, F., \& Moscardini, L. 1997, MNRAS, 286, 115

Moscardini, L., Coles, P., Lucchin, F., \& Matarrese, S. 1998, astro-ph/9712184

Padmanabhan, T. 1996, MNRAS, 278, L29

Peacock, J. A., \& Dodds, S. J. 1996, MNRAS, 280, L19

Peacock, J. A., \& Nicholson, D. 1991, MNRAS, 253, 307

Postman, M., Lauer, T., Szapudi, I., \& Oegerle, W. 1998, astro-ph/9804141

Shectman, S. A., Landy, S. Oemler, A., Tucker, D., Lin, H., Kirshner, R., \& Schechter, P. 1996, ApJ, 470, 172

Shepherd, C. W., Carlberg, R. G., Yee, H., \& Ellingson, E. 1997, ApJ, 479, 82

Sheth, R. K. \& Jain, B. 1997, MNRAS, 285, 231

Small, T. A., Ma, C.-P., Sargent, W. L. W., \& Hamilton, D. 1998, in preparation

Summers, F. J., Davis, M., \& Evrard, A. E. 1995, ApJ, 454, 1 


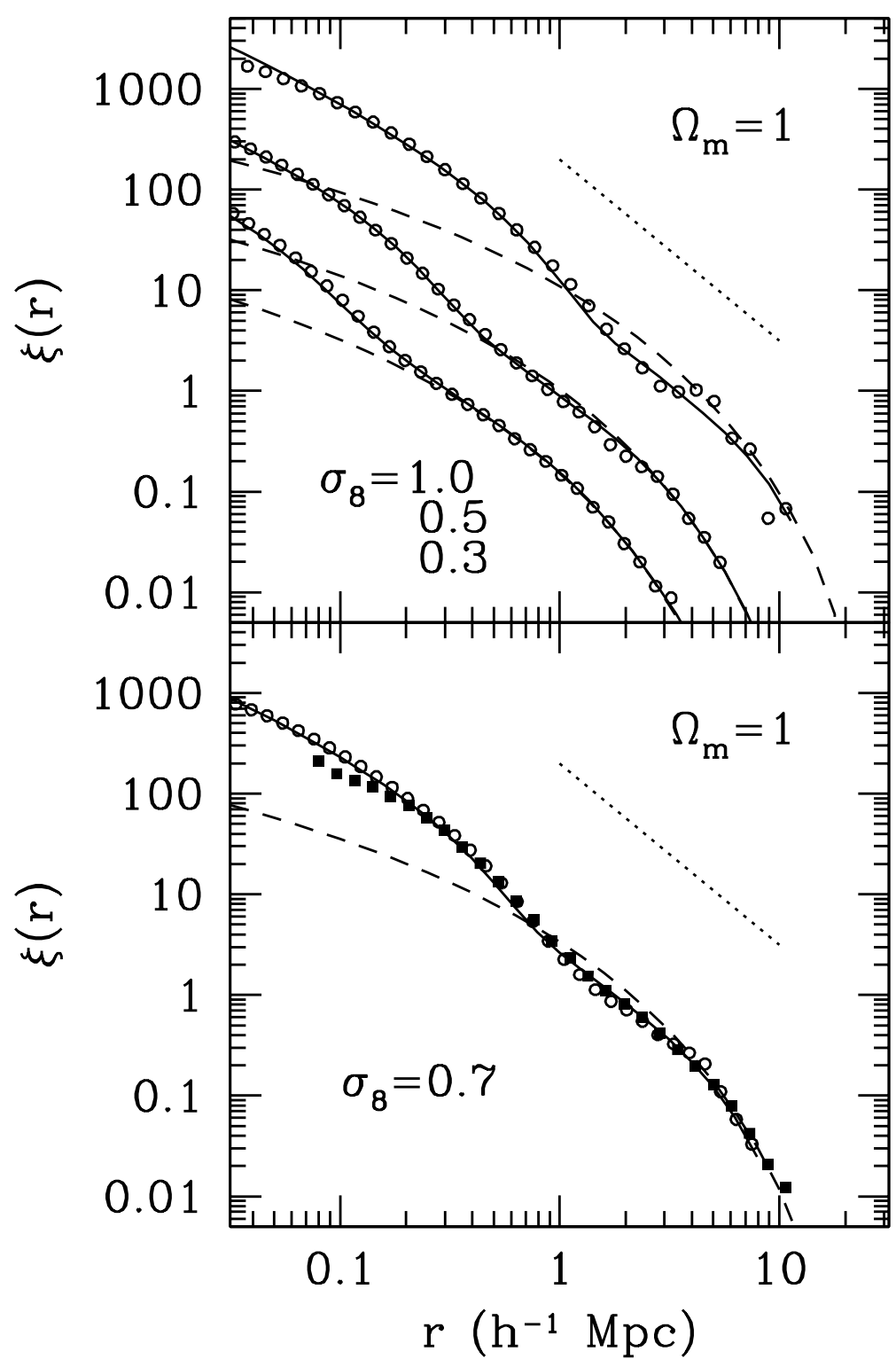

Fig. 1.- Evolution of the two-point correlation function $\xi(r)$ (in physical coordinates) for the matter density field in the standard CDM model with $\Omega_{\mathrm{m}}=1$ and $h=0.5$. The top panel shows results at $\sigma_{8}=0.3,0.5$, and 1.0 (from bottom up), and the bottom panel shows $\sigma_{8}=0.7$ separately for clarity. (The COBE normalization corresponds to $\sigma_{8}=1.4$ at $z=0$, while $\sigma_{8} \approx 0.7$ is required to match the observed abundance of $z \approx 0$ galaxy clusters.) The dashed curves show linear-theory predictions. The symbols represent the nonlinear $\xi$ computed from particles in the simulations the circles are from a simulation in a $(100 \mathrm{Mpc})^{3}$ box, and the solid squares for the $\sigma_{8}=0.7$ output is from a $(640 \mathrm{Mpc})^{3}$ simulation. The solid curves, which agree well with the symbols, represent the nonlinear $\xi$ from integration of the analytic nonlinear $P(k)$ in Ma (1998). The dotted straight line indicates a slope of -1.8 in this and the following figures. 


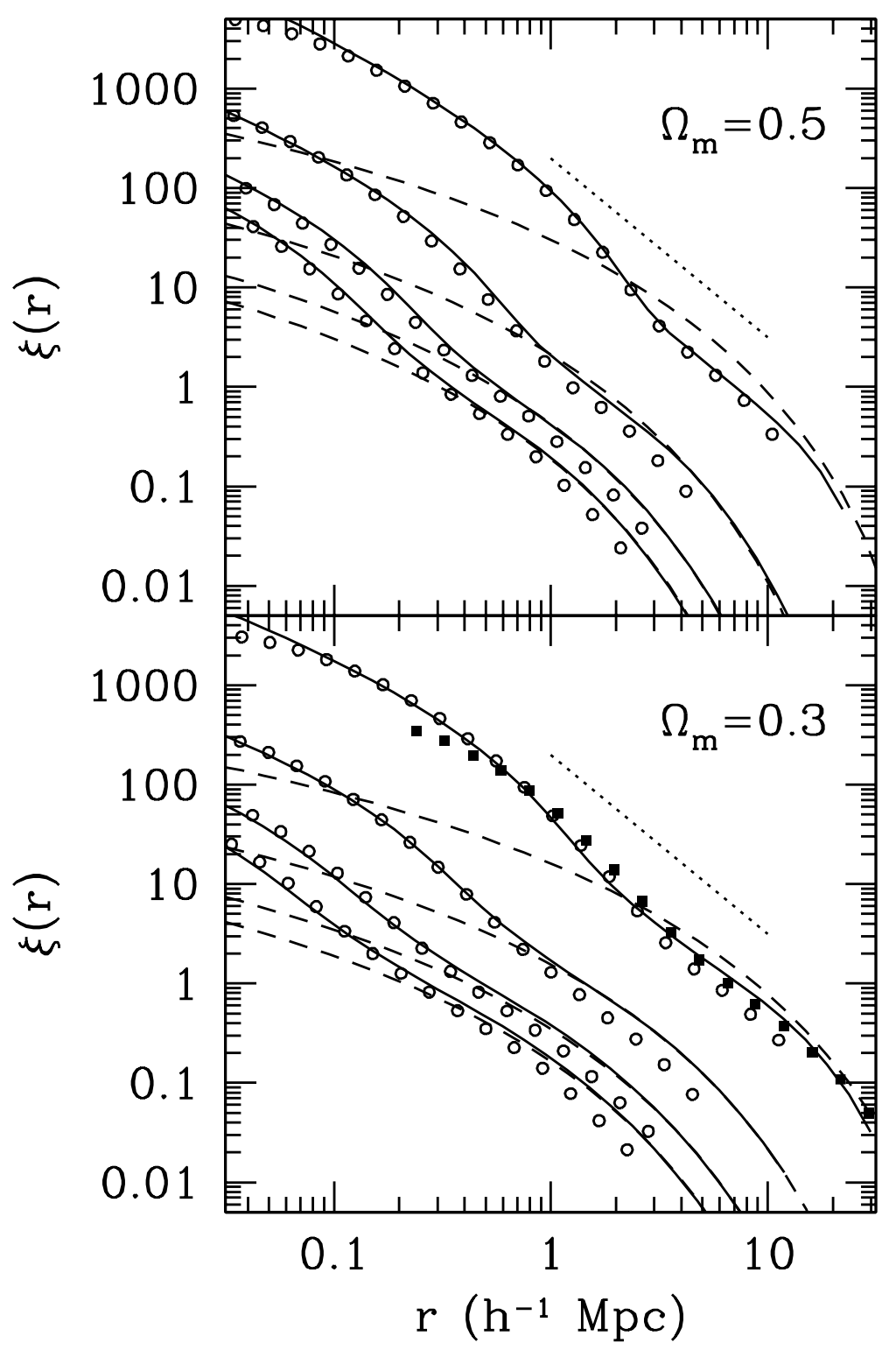

Fig. 2. - Same as Fig. 1 but for two LCDM models: $\Omega_{\mathrm{m}}=0.5, \Omega_{\Lambda}=0.5$, and $h=0.7$ (top); and $\Omega_{\mathrm{m}}=0.3, \Omega_{\Lambda}=0.7$, and $h=0.75$ (bottom). For each model, four redshifts are shown: $z=4$, $3,1.5$, and 0 (from bottom up). The solid squares compare $\xi$ computed from a large (640 Mpc) ${ }^{3}$ simulation of the $\Omega_{\mathrm{m}}=0.3$ model. 


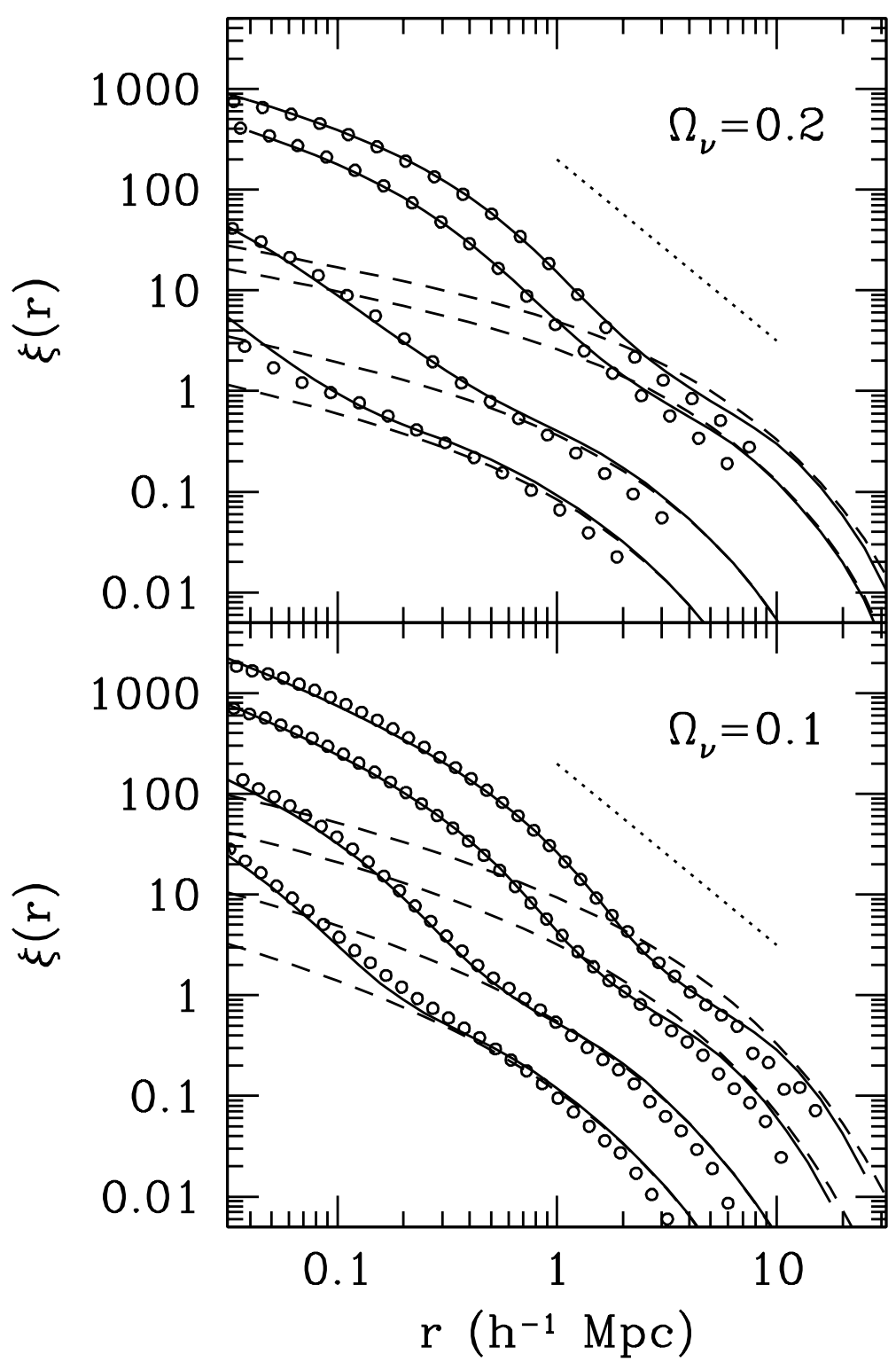

Fig. 3.- Same as Fig. 1 but for two C+HDM models: $\Omega_{\nu}=0.2, \Omega_{c}=0.75$ (top); $\Omega_{\nu}=0.1$, $\Omega_{c}=0.85$ (bottom). Both have $\Omega_{\mathrm{b}}=0.05$ and $h=0.5$. The redshifts shown are (from bottom up): $z=3,1.5,0.26$ and 0 for $\Omega_{\nu}=0.2$, and $z=3,1.5,0.43$ and 0 for $\Omega_{\nu}=0.1$. 


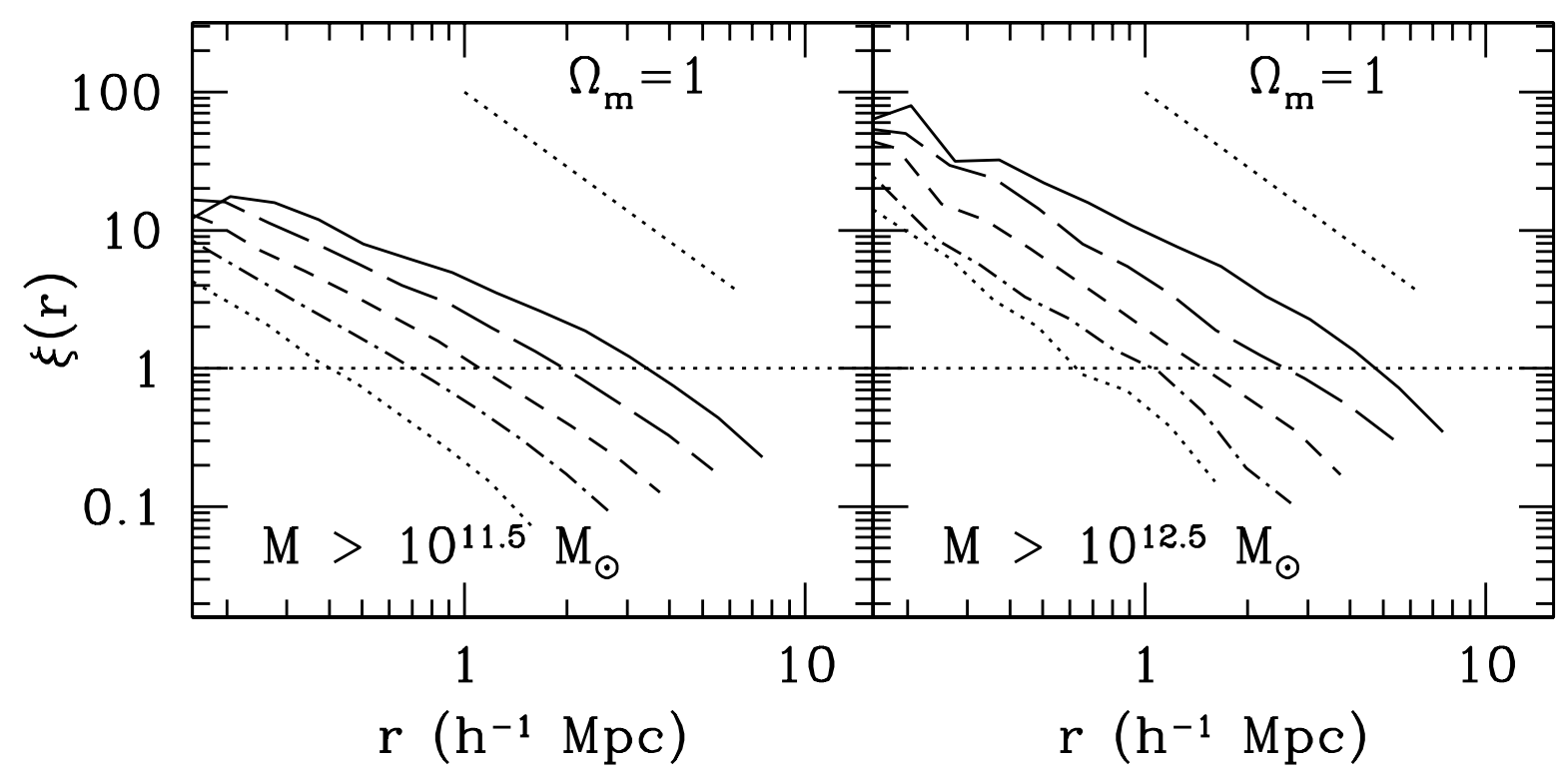

Fig. 4.- Evolution of the two-point correlation function $\xi(r)$ for the simulated dark matter halos in the standard CDM model with $\Omega_{\mathrm{m}}=1$ and $h=0.5$. Five epochs are shown: $\sigma_{8}=0.3$ (dotted), 0.5 (dot-short-dashed), 0.7 (short-dashed), 1.0 (long-dashed), and 1.4 (solid). (The COBE normalization corresponds to $\sigma_{8}=1.4$ at $z=0$.) The left and right panels show $\xi$ computed from virialized halos in the mass range $M>10^{11.5} M_{\odot}$ and $M>10^{12.5} M_{\odot}$, respectively. The dotted straight line indicates the observed slope of -1.8 . 

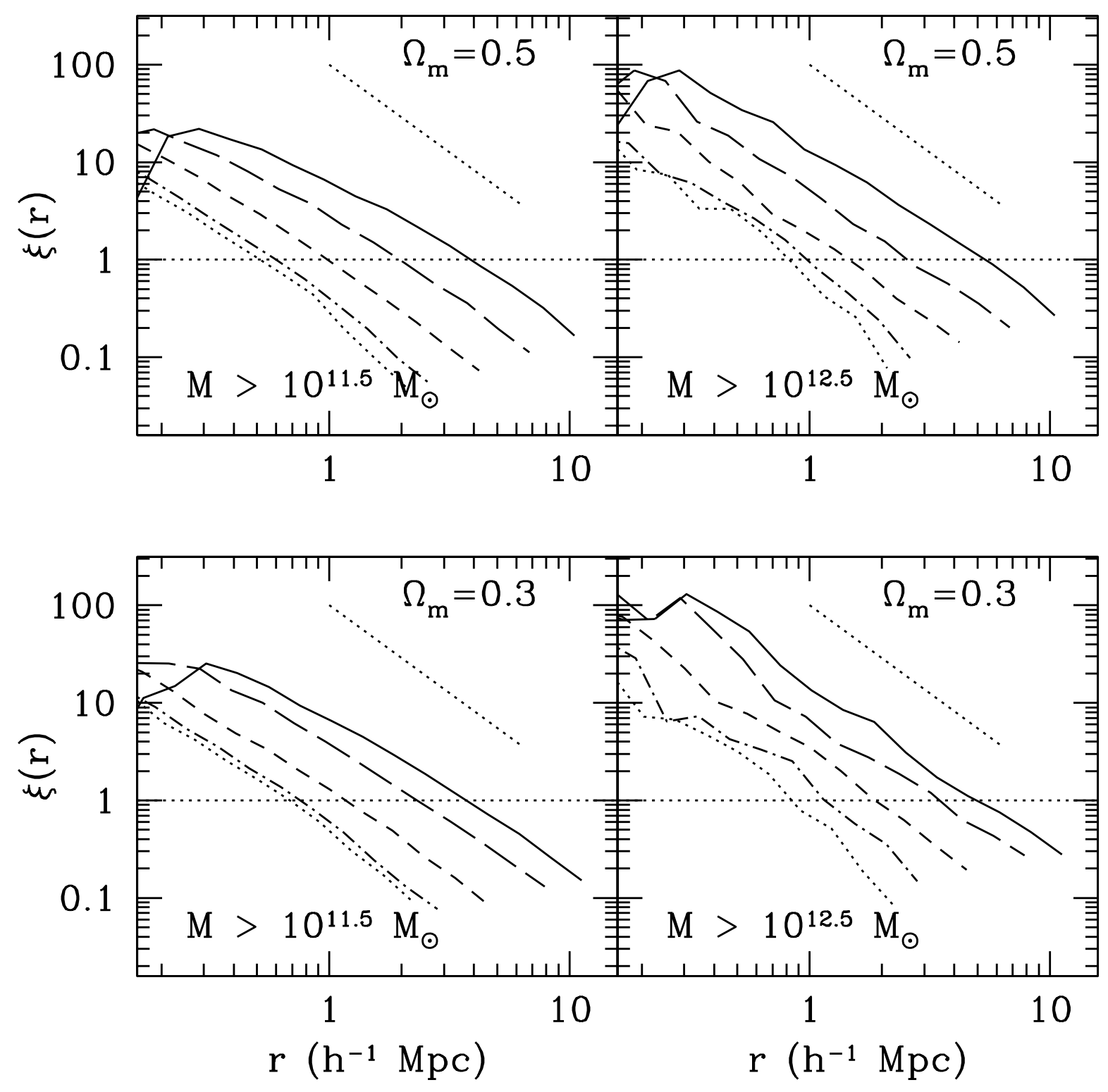

Fig. 5. - Same as Fig. 4 but for the two LCDM models: $\Omega_{\mathrm{m}}=0.5, \Omega_{\Lambda}=0.5$, and $h=0.7$ (top); and $\Omega_{\mathrm{m}}=0.3, \Omega_{\Lambda}=0.7$, and $h=0.75$ (bottom). Five epochs are shown: $z=4$ (dotted), 3 (dot-short-dashed), 1.5 (short-dashed), 0.5 (long-dashed), and 0 (solid). 

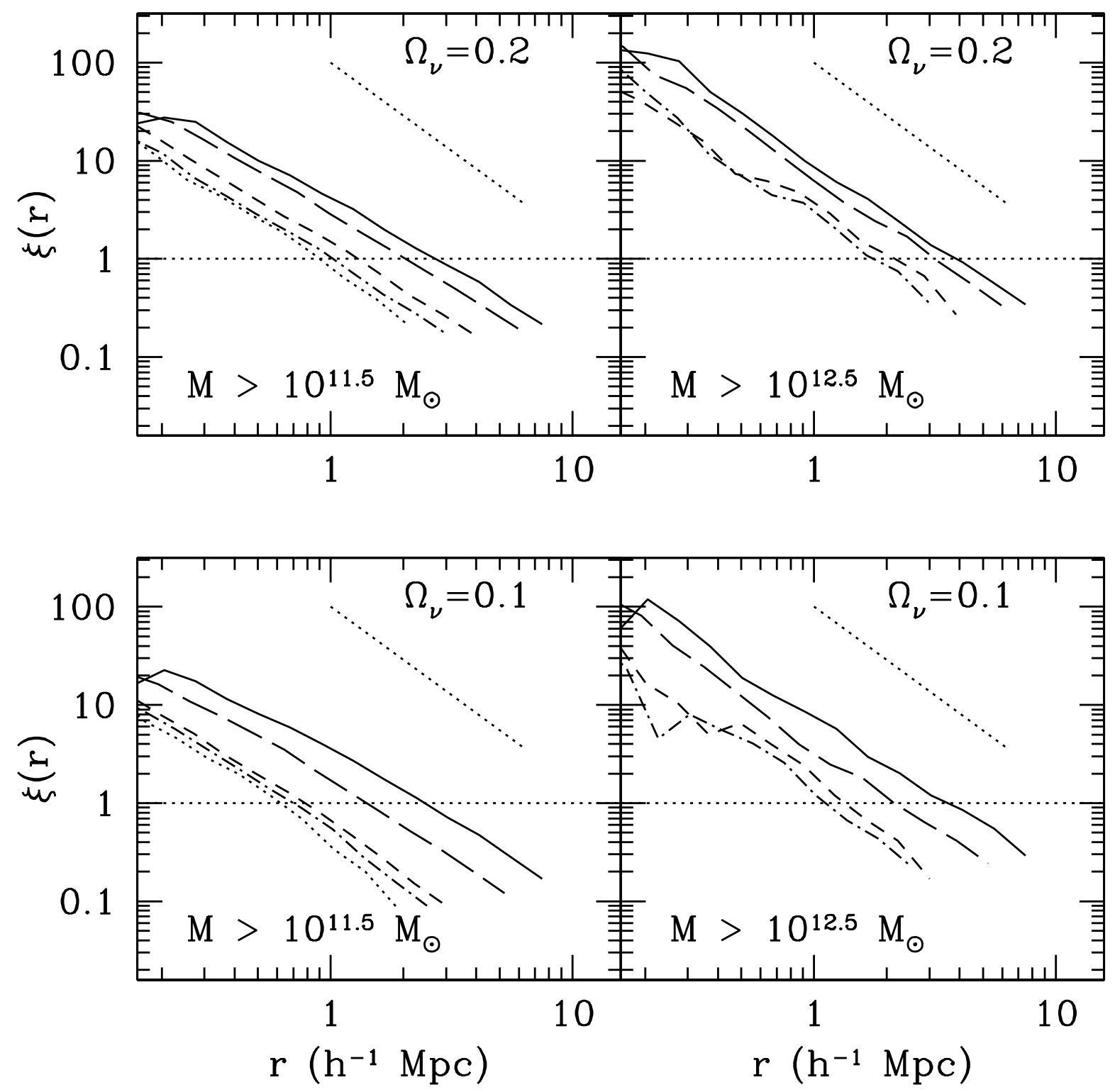

Fig. 6. - Same as Fig. 4 but for the two C+HDM models $-\Omega_{\nu}=0.2$ (top) and $\Omega_{\nu}=0.1$ (bottom). For $\Omega_{\nu}=0.2$, the redshifts shown are: $z=2.6$ (dotted), 1.5 (dot-short-dashed), 1.0 (short-dashed), 0.26 (long-dashed), and 0 (solid). For the $\Omega_{\nu}=0.1$ model, the redshifts are: $z=3$ (dotted), 2 (dot-short-dashed), 1.5 (short-dashed), 0.43 (long-dashed), and 0 (solid). (The dotted curves are omitted from the right panels since estimates of $\xi$ are very noisy due to the small number of massive halos at such early epochs formed in $\mathrm{C}+\mathrm{HDM}$ models.) 

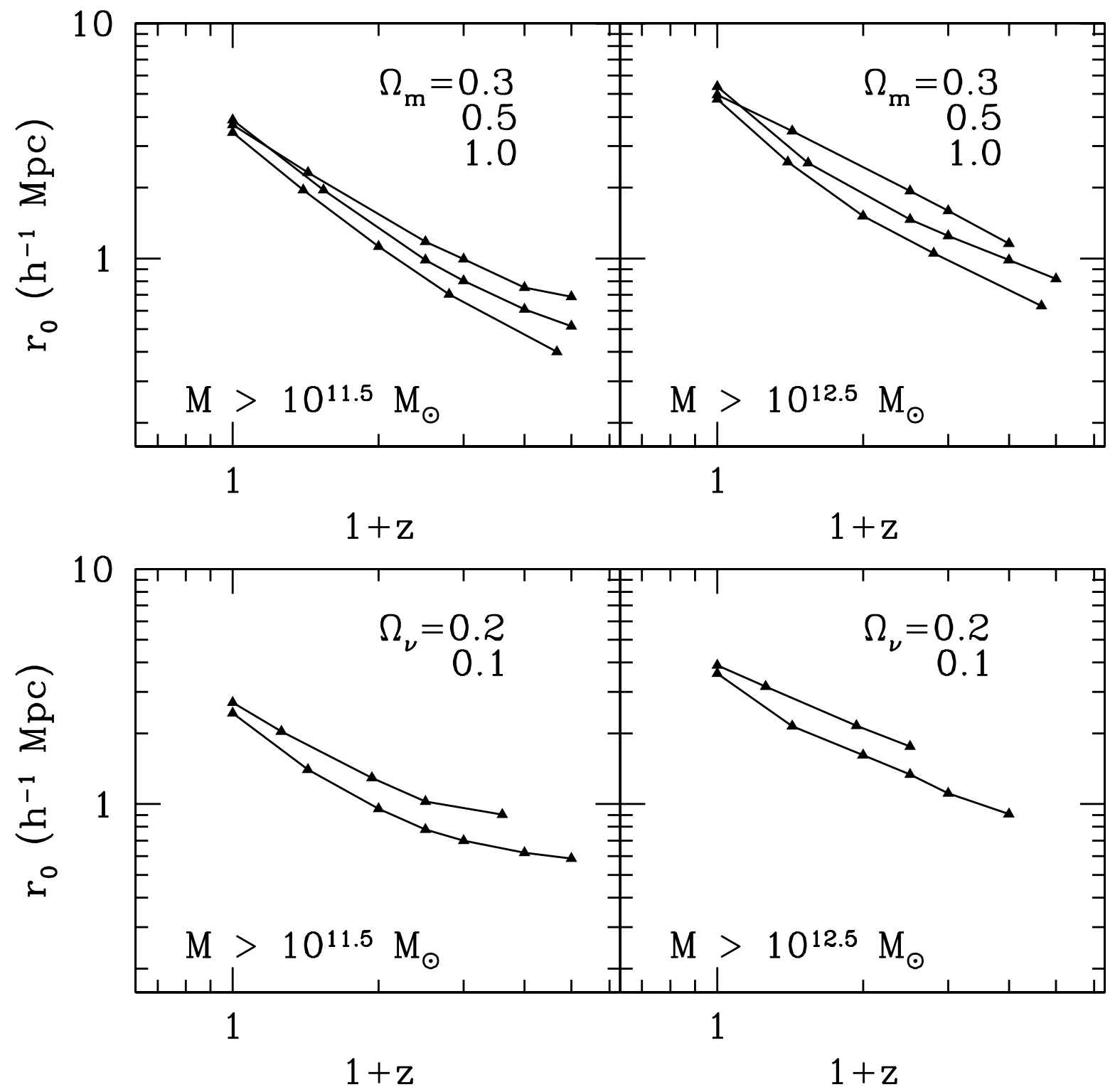

Fig. 7.- Redshift evolution of the physical correlation length $r_{0}$ for the simulated halos. In the top panels, the three curves from top down are for the $\Omega_{\mathrm{m}}=0.3,0.5$, and 1.0 LCDM models. In the bottom panels, the two curves are for the $\Omega_{\nu}=0.2$ (top) and 0.1 (bottom) C+HDM models. The redshift dependence of $r_{0}$ varies with cosmological parameters and is not a power law (see eq. [3]) as has often been assumed. The left and right panels compare two different halo mass ranges and show that more massive halos generally have a larger $r_{0}$. 


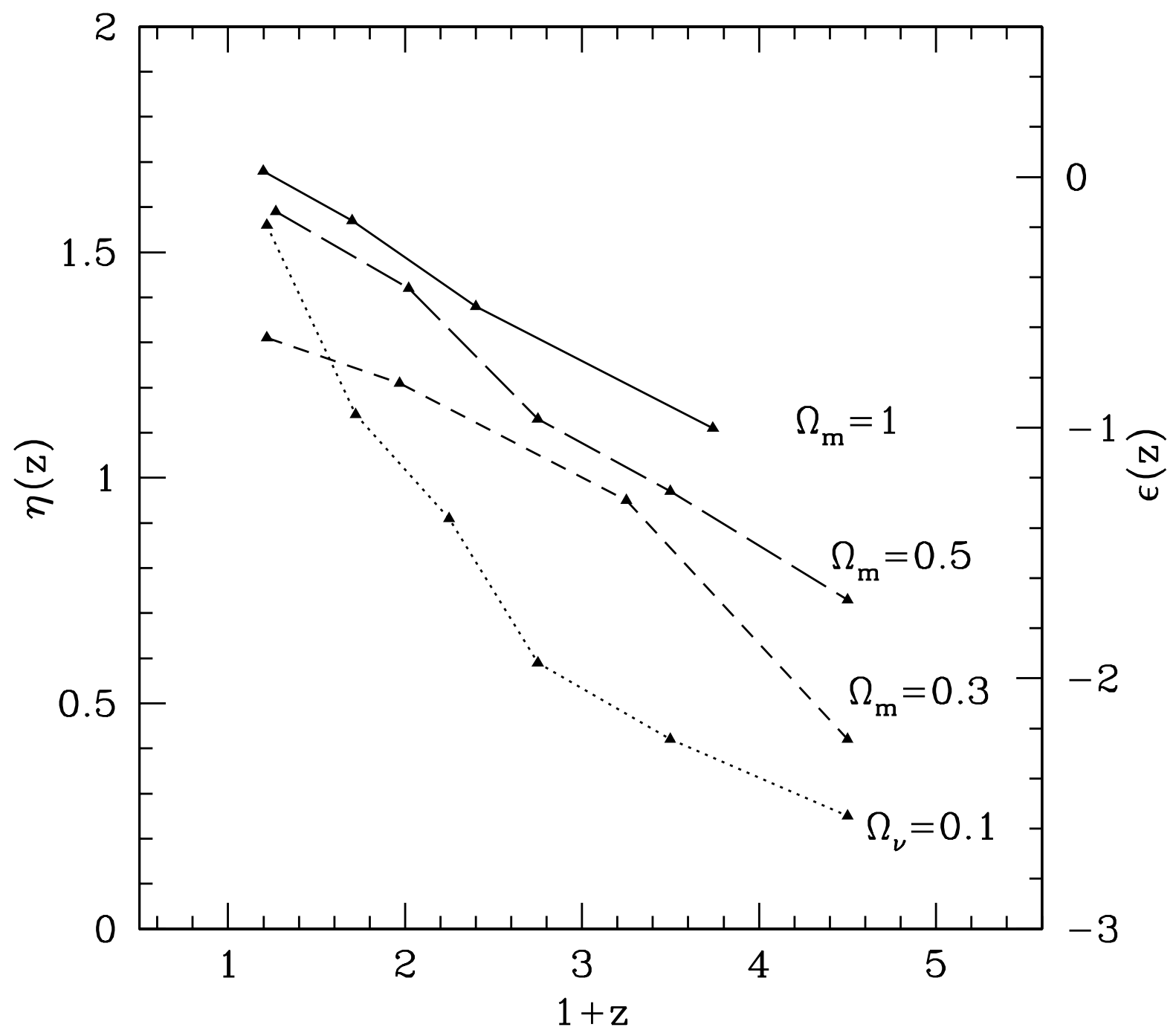

Fig. 8. - The evolution parameter $\eta(z)$, defined as $r_{0}(z)=(1+z)^{-\eta(z)} r_{0}(0)$, for the $M>10^{11.5} M_{\odot}$ virialized halos in four models: CDM, $\Omega_{\mathrm{m}}=0.5 \mathrm{LCDM}, \Omega_{\mathrm{m}}=0.3 \mathrm{LCDM}$, and $\Omega_{\nu}=0.1 \mathrm{C}+\mathrm{HDM}$ models. The COBE normalization is used, including the CDM. The vertical labels on the right mark the corresponding $\epsilon$ used in the literature, where slope of $\gamma=1.8$ has been assumed to relate $\eta$ and $\epsilon: \epsilon=1.8 \eta-3$ (see eq. [7]). The rise of $\eta$ with decreasing $z$ shows that the halo correlation length increases faster than a simple power law assumed in the literature. The clustering passes $\eta=1$ (i.e. $\epsilon=-1.2$ ) for fixed clustering in comoving coordinates and approaches $\eta=1.7(\epsilon=0)$ for stable clustering at $z \approx 0$. 